\title{
STUDY OF FLUID LAYER GRAVITY MOTION OVER VERTICAL SURFACE
}

\author{
Arestak Sarukhanyan \\ Department of Education Reforms ${ }^{1}$ \\ Norik Sarkisyan \\ Department of Architecture ${ }^{l}$ \\ Vache Tokmajyan \\ Department of Technology \\ Shushi University of Technology \\ 5 Mkhitar Gosh str., Stepanakert, Armenia, 375000 \\ Arevshad Vartanyan $\bowtie$ \\ Department of Management and Marketing of High-Tech Industries \\ Moscow Aviation Institute (National Research University) \\ 4 Volokolamskoe Highway, Moscow, Russia, 125993 \\ arevshadvartanyan@mail.ru \\ ${ }^{1}$ National University of Architecture and Construction of Armenia \\ 105 Teryan str., Yerevan, Armenia, 0009
}

$\triangle$ Corresponding author

\begin{abstract}
This paper presents the results of studying the motion of a liquid layer along the walls of a vertically installed pipe under the action of gravity. Two-dimensional boundary layer is formed by the fluid motion relative to the hard wall on surfaces of structures (pipes, turbines, heat-and-mass transfer equipment, aircrafts, ships, etc.), which are of positive interest in engineering practice. Further upgrading of the above-mentioned structures is possible only by increasing accuracy of momentum in the boundary layer, heat and mass transfer rates calculation. It is confirmed that in the boundary layer transfer phenomena intensity (perpendicular to the wall) is due to the fluid particles velocity distribution regularities in the cross-section of the layer. Fluid velocity distribution regularities in turn are conditioned by Reynolds number according to current notions. The principal method of quantitative analysis of turbulent flow in a boundary layer suggested by Reynolds continues to be the velocity and pressure fluctuations averaging method for some timespan. The suggested model of fluid movement enables to prognosticate conditions under which in cross-sections of the boundary layer reshaping of velocity profile takes place, to carry out analytic calculation of such hydrodynamic characteristics as mean velocity of motion, layer thickness and shearing stresses acting on the wall. The difference between the suggested methods developed for calculation of flow parameters from the well-known ones is in that that calculations are made based on an integrated approach regardless of such conceptual definitions as laminar and turbulent regimes widely used in modern hydrodynamics. Obtained results and design formulas known in the literature have been compared. It has been found that the thickness of the sliding layer, determine by the proposed calculation formula, 1.17 times smaller than that determined by the currently used formula.
\end{abstract}

Keywords: fluid, boundary layer, viscosity, velocity profile, mean velocity, shearing stresses.

DOI: $10.21303 / 2461-4262.2021 .002115$

\section{Introduction}

In the history of hydrodynamics development an important milestone was boundary layer notion introduced by [1]. According to its approaches fluid flow can be divided into two zones in the effective cross-section. Fluid viscosity has essential influence on a boundary layer being formed only near solid wall where the flow rate gradient is the greatest. In [2] boundary layer theory was developed which enables to reduce hydrodynamics equations without essential losses.

To study exchange phenomena in the boundary layer in the last century in a number of countries researchers carried out fundamental theoretical and experimental investigations [3]. Information on the study methods can be found in the following works [4, 5]. 
For the first time the idea of fluids motion by laminar and turbulent modes was advanced by Reynolds based on the results obtained after experimental study of a fluid flow in pipes of circular cross-section $[1,2]$.

Starting from a Reynolds number a fluid steady-state (laminar) flow in the form of layers is transformed to nonsteady-state chaotic (turbulent) flow.

In the current hydrodynamics modes of laminar and turbulent flow are studied as separate ones. Such an approach led to development of two theories of fluid flow which are of different content and investigation methods $[6,7]$. This situation can lead to serious mistakes in choosing calculation methods since neither theoretical nor experimental method does not yet allow predicting in advance the critical value of the Reynolds number at which a change of modes occurs [8,9].

The fluid laminar flow is analyzed by relatively not difficult analytical methods, which present no practical interest [2].

It is widely accepted that in turbulent flow the values of actual velocities of fluid particles motion is a sum of irregular velocity of various amplitudes and averaged velocity.

The averaging method of motion equations creates such additional difficulties as the increase of unknown quantities (of turbulent stresses) and the problem of equations number limitation.

The solution of this problems assumes establishment of connection between turbulent stresses (Reynolds stresses) and deformation tensors of averaged velocities.

To get a closed mathematical problem, usually, specific turbulence models are used which have no physical justifications [8].

Complexity of fluid turbulent flow mechanisms revelation is conditioned by determination of the transfer factor (of turbulent viscosity) value. To simplify the problem Boussinesq was the first to suggest the idea of turbulent viscosity, which was further developed in [8]. To calculate the turbulent viscosity Prandtl factor based on the molecular transport momentum analogue suggested a hypothesis $[2,8]$ on the size of turbulent streams mixing path. Afterwards Carman making use of the theory of dimension methods for calculation of turbulent viscosity suggested another approach [9].

So far physical justification methods for calculation of the transport factor magnitude (turbulent viscosity) of momentum in turbulent stream have not been developed.

All known semi-empirical approaches on turbulent transport do not enable to obtain an idea of dependency of averaged velocity and parameters of irregular nature.

The question of averaged velocity distribution law for the turbulent boundary layer cross-section continues to remain problematic.

Based on subjective perceptions on momentum transport mechanisms in different zones of the turbulent boundary layer different researchers suggest different regularities for the flow averaged velocity distribution. According to Prandtl hypothesis distribution of the flow averaged velocity in the sublayer located next to the wall occurs by linear law which then was called laminar or viscose sublayer [10]. According to a scheme suggested by Cramer near the wall the turbulent flow is divided into three zones:

1) laminar zone of the boundary layer;

2) «buffer» zone;

3) turbulent flow zone $[11,12]$. It is assumed that in the region of turbulent flow which occupies the main part of the flow, averaged velocities are distributed by logarithmic or exponential laws.

The problem first became the subject of a detailed study by [3]. Later on [8] generalized the obtained results for viscous fluids. In [1] to study stability of fluids motion carried out important research.

Numerical simulation of the Navier-Stokes equations enables to obtain solutions of some problems of utmost importance for explaining laminar-turbulent transformations in gases and fluids flows of different nature [13].

Different models exist designed to explain laminar-turbulent modes, transformations of which information on the recent advances of that time is outlined in the monograph [14].

All known models are empirical and are not universal at that each one has its application limited range. Reasons of liquid stream laminar-turbulent modes transformation phenomenon until now have not been explained. 
To solve averaged Navier-Stokes equations according to the Reynolds (RANS-Reynolds Averaged Navier-Stokes) different approaches have been developed. Starting from 1970-1980s researchers began to use asymptotic methods of study [15].

Beginning from 1990s due to rapid development of computer engineering it became possible to conduct research of fluids flow based on numerical modeling techniques.

To calculate turbulent motion characteristics programs currently are drawn up mainly by Reynolds averaged equations (for example, Ansys CFX, Ansys Fluent, Star-CD, LOGOS, etc.).

Problems related to impulse, heat and mass combined transfer formed in boundary layers under different hydrodynamic conditions continue to remain at the center of researchers' attention [16-18].

Many works are devoted to research of the boundary layer formation on axisymmetric bodies, particularly, when it is formed upon a cylinder along its axis under streamlined liquid. It has been substantiated that when the boundary layer thickness is smaller than the cylinder radius the curvature of cross-section can be neglected, that is the boundary layer formed on the cylindrical surface is no different from a boundary layer formed on a plane.

\section{Materials and methods}

Under the influence of gravity the formation of fluids flow in the form of a thin layer found widespread application in heat-and-mass transfer equipment which are widely used in power engineering, chemical and food industries to increase intensity of transfer phenomena.

To study momentum, heat-and-mass transfer mechanisms occurring in sliding fluid numerous experimental and theoretical studies have been carried out.

It is well known that gravitational motion of a fluid film along a vertical wall occurs in several modes [10]. Laminar flow of the fluid film when on its free surface there are no waves, the Reynolds number values are $\operatorname{Re}_{L}<30 \div 50$, laminar motion with surface waves is observed under $30<R e_{L}<400$ conditions, and when $R e_{L}>400$ the motion is turbulent. For the fluid film the Reynolds number is determined by $\operatorname{Re}_{L}=G / \mu$, where $G=\rho \cdot u \cdot \delta$ is the fluid flow for the unit width of the fluid film, $u$ is the mean velocity of the fluid film, $\delta$ is the thickness of the film, $\rho$ is the fluid density, $\mu$ is the dynamic viscosity index. The laminar motion of the fluid film under influence of the force of gravity over a flat surface for the first time was considered by [19]. The problem of the layer turbulent motion later on was studied by [10]. The problem of motion in conditions of waves' development on the free surface of the fluid film was first posed by [20].

\section{1. The aim and objectives of the study}

Problems of transfer phenomena running in the sliding fluid layer under the influence of gravity have not been exhaustively studied to date and still remain in the center of researchers' attention:

- to suggest theoretically grounded and in engineering practice applicable new methods for calculation of hydrodynamic characteristics of the fluid film sliding along a vertical wall under influence of the gravity force;

- to show possibility of calculation of a sliding layer hydrodynamic parameters from a unified viewpoint not connecting with notions of laminar and turbulent motion modes as they are perceived in current hydrodynamics.

The work is based on approaches described in papers [21] and it is their extension. There are suggested theoretically grounded and in engineering practice applicable new methods for calculation of hydrodynamic characteristics of the fluid film sliding along a vertical wall under influence of the gravity force.

There is shown the possibility of calculation of sliding layer hydrodynamic parameters from a unified viewpoint not connecting with notions of laminar and turbulent flow modes as they are perceived in current hydrodynamics.

\section{2. Calculation methods and obtained results}

Motion of an incompressible viscous fluid film under influence of gravity force over the wall of a vertically installed pipe of circular cross-section under isotherm and steady-state conditions, at that free surface of the film is flat. 
Within the boundary layer theory approximation fluid film motion and flow continuity equations are given by [21]:

$$
\begin{gathered}
u \frac{\partial u}{\partial z}+v \frac{\partial u}{\partial r}=g+v \frac{\partial^{2} u}{\partial r^{2}} \\
\frac{\partial u}{\partial z}+\frac{\partial v}{\partial r}=0
\end{gathered}
$$

in the case when there are the following conditions:

$$
\delta \ll R, \frac{\partial p}{\partial z}=0, \frac{\partial^{2} u}{\partial z^{2}} \ll \frac{\partial^{2} u}{\partial r^{2}},
$$

where $z$ axis is directed along the wall of the pipe, $r$ is the distance from the wall, $R$ is the radius of the pipe, $\delta$ is the actual thickness of the layer, $v=\mu / \rho$ is the momentum transfer factor, $\mu$ is the fluid viscosity factor, $\rho$ is the density, $g$ is free fall acceleration, $u, v$ are the velocity components directed along $z$ and $r$ axes, respectively.

Transfer phenomena intensity in the boundary layer being formed near a hard wall is conditioned by hydrodynamic conditions of the layer formation that is by what law potential flow velocity is changed along the wall.

To solve equations (1), (2) boundary conditions usually are given by:

$$
\begin{aligned}
& u=v=0, \text { when } r=0, \\
& u \rightarrow U_{0}, \text { when } r \rightarrow \delta_{0},
\end{aligned}
$$

where $U_{0}=\sqrt{2 g z}$ is the velocity of potential flow, $\delta_{0}$ is the thickness of the boundary layer.

It is important to note that the magnitude of transfer momentum $v$ factor (kinematic factor of viscosity) in the course of the problem solution is accounted constant and is not conditioned by modes of the fluid motion.

By means of the below dimensionless functions:

$$
\begin{gathered}
\eta=\frac{1}{\sqrt{2}} \cdot r\left(\frac{2 g}{v^{2}}\right)^{1 / 4} \cdot \frac{1}{z^{1 / 4} 14}, \\
f(\eta)=\frac{1}{\sqrt{2}} \cdot \frac{\psi(z, r)}{\left(\frac{2 g}{v^{2}}\right)^{1 / 4} \cdot v \cdot z^{3 / 4}}
\end{gathered}
$$

where $\psi(z, r)$ is the flow function, differential (1), (2) with partial derivatives are transformed into dimensionless of one third order ordinary differential equation for the flow function:

$$
f^{\prime \prime \prime}+\frac{3}{2} f f^{\prime \prime}-\left(f^{\prime}\right)^{2}+1=0
$$

The boundary conditions expressed by new parameters are:

$$
\begin{gathered}
f=f^{\prime}=0 \text { when } \eta=0, \\
f^{\prime} \rightarrow 1 \text { when } \eta \rightarrow \eta_{0},
\end{gathered}
$$

where $f^{\prime}=u / U_{0}$ is the dimensionless distribution function of velocities, $\eta_{0}$ is the dimensionless thickness of the boundary layer. 
Designating the magnitude of $f^{\prime \prime}$ function on the wall $f_{0}^{\prime \prime}=b$, approximate solution of (6) for small quantity of $\eta$ can be expressed in the form of the following series:

$$
f=\frac{b}{2 !} \eta^{2}-\frac{1}{3 !} \eta^{3}+\frac{b}{4 \cdot 6 !} \eta^{6}-\ldots
$$

In case of (7) boundary conditions by integration of (6) using numerical methods [22]:

$$
b=f_{0}^{\prime \prime}=1.272 .
$$

Peculiarity of the flow $f$ function is that at a small distance from the wall it is changed by quadratic law, for larger quantities of $\eta$ the curve gradually is straightening and asymptotically tends to the bisector of an angle made by coordinate axes.

Depending on variation range magnitude of $\eta$, starting from coordinate system origin, solution of (8) enables to represent velocity distribution laws in the boundary layer by different approximations.

It follows from evaluation of (8) terms that $\eta$ parameter's variation within the $0<\eta<0.3$ small range the solution can be confined to the first term of the above series and accept:

$$
f \approx \frac{b}{2 !} \eta^{2}
$$

The absolute value of error is:

$$
\left|R_{1}(\eta)\right| \leq \frac{1}{3 !} \eta^{3}=4.5 \cdot 10^{-3}(\text { when } \eta=0.3)
$$

When parameter $\eta$ varies within $0<\eta<0.3$ to describe the behavior of $f$ function of the flow the solution can be confined to the two terms of the above series and accept:

$$
f \approx \frac{b}{2 !} \eta^{2}-\frac{1}{3 !} \eta^{3}
$$

The absolute value of error is:

$$
\left|R_{2}(\eta)\right| \leq \frac{b}{4 \cdot 6 !} \eta^{6}=3.32 \cdot 10^{-3}(\text { when } \eta=1.4)
$$

To simplify further calculations it is convenient to approximate equation (10) by the below exponential function:

$$
f(\eta) \approx 0.47 \eta^{1.788}
$$

In cross-sections of the fluid layer sliding over the wall the shape of the velocity distribution function is conditioned by the thickness of the fluid layer. When the layer thickness varies within $0<\eta<0.3$, the velocity distribution function according to equation (9) is:

$$
f^{\prime}=\frac{u}{u_{e}}=f_{0}^{\prime \prime} \cdot \eta
$$

where $u_{e}=U_{0} \cdot f^{\prime}(\eta)$ is the velocity of the layer free surface motion.

From equation (12) it follows that in cross-sections of thin films longitudinal components of velocities of fluid particles motion depending on the distance from the wall are distributed 
according to the below quadratic law: $u=u_{e} \cdot f_{0}^{\prime \prime} \cdot \eta=U_{0} \cdot\left(f_{0}^{\prime \prime}\right)^{2} \cdot \eta^{2}$. The flow being formed within this range of the boundary layer is generally accepted to call viscous sublayer.

In case of large flows when the thickness of the fluid layer exceeds the thickness of the viscous sublayer self-regulation of the flow occurs and in the cross-section, according to (11) (when $\eta$ parameter varies within $0<\eta<1.4$ ) the function of velocity distribution can be approximated by:

$$
f^{\prime}=\frac{u}{u_{e}} \approx 0.84 \cdot \eta^{0.788}
$$

where the velocity of the layer free surface motion is determined by:

$$
u_{e}=0.84 U_{0} \eta^{0.788} .
$$

Therefore, longitudinal components of velocities in the layer cross-section are distributed according to $u \sim \eta^{1.576}$ exponential law.

According to equation (14), when $\eta=1.4$, then from $f^{\prime}=u_{e} / U_{0} \approx 1.09$ and equation (7) boundary conditions, the second is quite acceptable for engineering calculations.

To be sure that functions ((12), (13)) obtained for velocity distribution in the boundary layer are reliable let's make calculations to determine values of hydrodynamic parameters of flow.

To make further calculations it is advisable to represent (4) in the below form:

$$
\eta=\frac{1}{\sqrt{2}} \cdot R e^{1 / 2} \cdot \frac{1}{z} \cdot \frac{q}{<u>}
$$

where $\operatorname{Re}=\left(U_{0} \cdot z\right) / \mathrm{v}$ is the Reynolds longitudinal number calculated according to the length of the pipe, $\langle u\rangle$ is the mean velocity of the layer motion, $q=\delta \cdot\langle u\rangle$ is the value of the volume flow per unit width of the layer.

In the cross-section of a layer located at an arbitrary $z$ distance from the starting point of the pipe the mean value of velocity of the fluid particles motion along $z$ depending on the layer thickness is determined by the below formula:

$$
<u>=\frac{1}{\delta} \int_{0}^{\delta} u \mathrm{~d} r=\frac{1}{\eta} \int_{0}^{\eta} u_{e} f^{\prime}(t) \mathrm{d} t .
$$

Taking into consideration equation (12), let's obtain:

$$
<u>=\frac{1}{3} U_{0}\left(f_{0}^{\prime \prime}\right)^{2} \eta^{2}
$$

where substituting into equation (15):

$$
\eta^{3}=\frac{3}{\sqrt{2}\left(f_{0}^{\prime \prime}\right)^{2}} \cdot \frac{q}{z \cdot U_{0}} \cdot R e^{1 / 2} .
$$

From this it follows:

$$
\eta=\left(\frac{3}{4 \sqrt{2}\left(f_{0}^{\prime \prime}\right)^{2}}\right)^{1 / 3} 13 \frac{R e_{L}^{1 / 3}}{R e^{1 / 6}}=0.69 \cdot \frac{R e_{L}^{1 / 3}}{R e^{1 / 6}},
$$

where $R e_{L}=4 q / v$ is the Reynolds number.

(18) enables to directly represent regularities of velocity distribution and the actual thickness of the layer through Reynolds $\operatorname{Re}_{L}$ and $R e$ criteria. 
Real thickness of the layer depending on the flow value is determined by (4), (18).

$$
\delta=0.974 \frac{R e_{L}^{1 / 3}}{R e^{2 / 3}} \cdot z=0.773 \frac{R e_{L}^{1 / 3} \cdot v^{2 / 3}}{g^{1 / 3}} .
$$

According to (19) in case of small flow, when the fluid layer thickness varies within the range of viscous sublayer, the real thickness of the layer is conditioned only by the value of the Reynolds $\operatorname{Re}_{L}$ number and remain constant along the flow for the same flow.

In addition (18) enables to evaluate the thickness of the viscous sublayer. If it is assumed that in the cross-section rearrangement of longitudinal components of velocities occurs, when $\eta_{k r}=0.3$, then according to equation (16) the value of the Reynolds $\mathrm{Re}_{L k r}$ number is determined by:

$$
R e_{L k r}=0.082 R e^{1 / 2}
$$

It follows from (20) that along the flow in different cross-sections of the viscous sublayer the Reynolds $R e_{L k r}$ critical number has different values depending on the Reynolds (Re) number along the length.

The thickness of the viscous sublayer depending on the length and physical properties of the pipe, and taking into account equation (19), (20), is changed by the below law:

$$
\delta_{k r}=\frac{0.423}{R e^{1 / 2}} \cdot z
$$

That is the thickness of the viscous sublayer along the wall increases by $\delta_{k r} \sim z^{1 / 4}$ law.

Values of local shearing stresses on the wall of the pipe are determined by the below equation:

$$
\tau_{0}=\left.\mu \frac{\partial u}{\partial r}\right|_{r=0}=\left.\mu \frac{\partial u}{\partial \eta} \cdot \frac{\partial \eta}{\partial r}\right|_{\eta=0}=\frac{2}{\sqrt{2}} \mu \cdot U_{0} \cdot\left(f_{0}^{\prime \prime}\right)^{2} \cdot\left(\frac{2 g}{v^{2}}\right)^{1 / 4} 14 \cdot \frac{1}{z^{1 / 4}} \cdot \eta .
$$

Here substituting equation (18) and making appropriate transformations:

$$
\tau_{0}=1.583 \cdot \rho \cdot U_{0}^{2} \frac{R e_{L}^{1 / 3}}{R e^{2 / 3}}=1.583 \cdot(2 g v)^{2 / 3} 23 \cdot R e_{L}^{1 / 3} .
$$

It follows from equation (23) that when the fluid moves in the viscous sublayer range, values of local shearing stresses on the wall does not depend on the pipe length.

The value of friction coefficient is determined by:

$$
c_{w}=\frac{2 \tau_{w}}{\rho U_{0}^{2}}=3.166 \cdot \frac{R e_{L}^{1 / 3}}{R e^{2 / 3}} .
$$

Under the fluid large flow condition when in the cross-section the real $\delta$ thickness exceeds critical $\delta_{k r}$ thickness of the viscous sublayer $\delta>\delta_{k r}$, velocity distribution in the cross-section corresponds to (13) law.

Calculations of the motion hydrodynamic characteristics are made by the same analogy.

The mean velocity of the fluid motion is determined by:

$$
<u>=\frac{1}{\delta} \int_{0}^{\delta} u \mathrm{~d} r=\frac{1}{\eta} U_{0} \int_{0}^{\eta}\left[f^{\prime}(t)\right]^{2} \mathrm{~d} t=0.274 U_{0} \eta^{1.576} .
$$

To determine dimensionless thickness of the layer the below expression is used:

$$
\eta=\frac{1}{\sqrt{2}} \frac{R e^{1 / 2}}{z} \frac{q}{<u>}=0.844 \cdot \frac{R e_{L}^{0.388}}{R e^{0.194}}
$$


And the real thickness of the layer is determined by:

$$
\delta=1.19 \cdot \frac{R e_{L}^{0.388}}{R e^{0.694}} \cdot z
$$

According to equation (27) under the same flow conditions the layer thickness along the wall decreases by $\delta \sim l^{-0.325}$ regularity.

Local shearing stresses on the wall are determined by:

$$
\tau_{0}=\left.\mu \frac{\partial u}{\partial \eta} \frac{\partial \eta}{\partial r}\right|_{\eta=0}=0.801 \cdot \mu \cdot U_{0} \cdot R e^{1 / 2} \frac{1}{z} \cdot \eta^{0.576}
$$

The average value of shearing stresses along the wall is determined by:

$$
\tau_{w}=\frac{1}{l} \int_{0}^{l} \tau_{0}=0.671 \cdot \rho \cdot U_{0}^{2} \cdot \frac{R e_{L}^{0.224}}{R e^{0.612}} .
$$

According to equation (29) friction coefficient is determined by:

$$
c_{\varpi}=\frac{2 \tau_{w}}{\rho U_{0}^{2}}=1.342 \cdot \frac{R e_{L}^{0.224}}{R e^{0.612}} .
$$

According to the results obtained by calculations based on (23) and (29), at the moment of the motion mode transformation (when $\eta_{k r}=0.3$ ), shearing stresses rate on the pipe wall decreases approximately 1.25 times.

\section{3. Experimental procedures}

To calculate the thickness of fluid layer, formed in various hydrodynamic conditions, the authors of this work find it effective to plot (Fig. 1) the (19), (27) and compare them with the known in the literature equations.

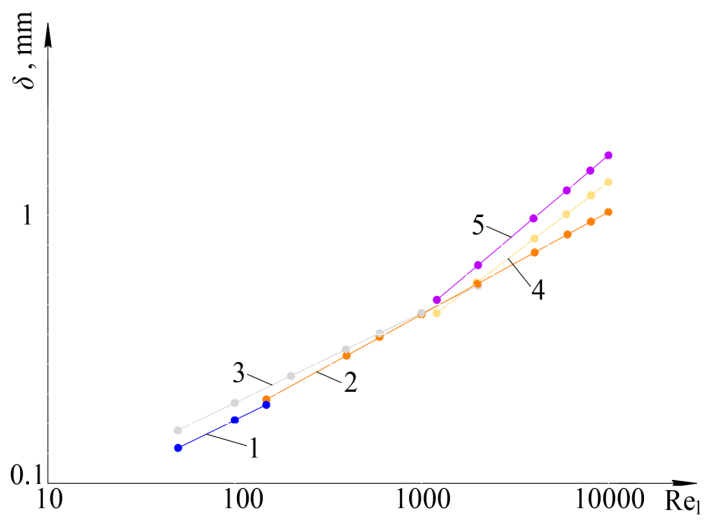

Fig. 1. The layer thickness graphs depending on the Reynolds number are plotted according to:

$$
1 \text { - Eq. (32); } 2 \text { - Eq. (27); } 3 \text { - Eq. (31); } 4 \text { - Eq. (33); } 5 \text { - Eq. (34) }
$$

Calculations have been performed for $l=1 u$ and $v=1 \cdot 10^{-6} \mathrm{~m}^{2} / \mathrm{s}$. In practically applicable limits of the Reynolds number the average deviation is $\pm 8 \%$.

Shearing stresses acting on the wall usually are calculated based on equilibrium conditions of forces acting on an element of the layer [13, 23]. 
In different modes of flow for calculation of shearing stresses (23) and (29) proposed in this work are graphically (Fig. 2) compared with known equations.

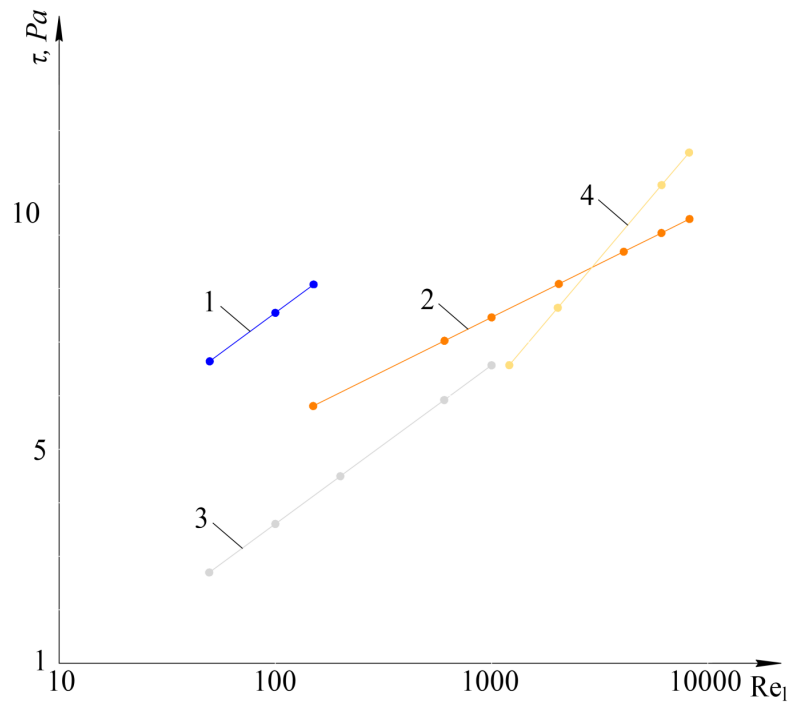

Fig. 2. Shearing stress graphs depending on the Reynolds number are plotted according to: 1 - Eq. (23); 2 - Eq. (29); 3, 4 - according to the results obtained by the authors [23]

\section{Results and discussion}

To develop a unified calculation mathematical model of the flow hydrodynamic parameters dimensionless parameters (4) and (5) were introduced by which the equations were reduced to the third order simple integral equation (6). The obtained equation was integrated for different sublayers case of the prescribed boundary conditions. As a result, to determine hydraulic parameters of different sublayers, calculation formulae were derived.

Particularly, to calculate the fluid layer thickness under laminar flow $\operatorname{Re}_{L}<1200$ authors suggest Nusselt approximate equation:

$$
\delta=0.908 \cdot \theta \cdot R e_{L}^{1 / 3}
$$

where $\theta=\left(v^{2} / g\right)^{1 / 3}$ is reduced thickness of layer.

To determine the layer thickness under $\operatorname{Re}_{L}<R e_{L k r}$ conditions the authors suggest equation (19) which after appropriate transformations can be written as:

$$
\delta=0.773 \cdot \theta \cdot R e_{L}^{1 / 3}
$$

The values of the layer thickness calculated by the Nusselt formula (31) are 1.17 times higher than the values calculated by the proposed formula (32).

Assuming that in turbulent mode $R e_{L}>1200$ velocities in the layer are distributed by logarithmic law, authors $[22,24]$ calculate the layer thickness suggest the following equation:

$$
\delta=0.208 \cdot \theta \cdot R e_{L}^{8 / 15}
$$

Authors [23] assuming that in turbulent flow velocities are distributed according to Blasius 7/12 exponential law, to calculate the layer thickness set up the below equation:

$$
\delta=0.37 \frac{R e^{7 / 12} \cdot v^{2 / 3}}{g^{1 / 3}}=0.165 \theta R e_{L}^{7 / 12} .
$$

Here it was denoted as $R e=q / \mathrm{v}$. 
The present study relates to the two-dimensional boundary layers. It should be noted that this problem is of important practical application. However, the two-dimensional boundary layer is a partial solution of the problem. Hence, problems related to boundary layers require additional research. To expand limits of the results of the present study it is necessary to turn to thee-dimensional problems, which from mathematical point of view it is difficult. Finite elements technique is the right key to solve such problems.

\section{Conclusions}

The suggested model of sliding incompressible and viscous fluid motion under the action of gravity force enables to calculate hydrodynamic characteristics from a uniform point of view that is without connecting with laminar and turbulent modes notions. In the course of calculations the momentum transfer factor magnitude (viscosity of fluid) is considered as constant magnitude. It is shown that depending on fluid physical properties, velocity of flow and potential flow, fluid motion in the boundary layer range can take place by two different laws of velocity distribution. In case of small flows, when flow occurs within the $\eta<\eta_{k r}=0.3$ range of the boundary layer (in viscous layer), velocity profile in the cross-section of the layer is formed by parabolic law.

In cross-section of the boundary layer, if the thickness of the fluid layer exceeds the thickness of the viscous sublayer, a self-regulation is observed, that is redistribution of the velocity profile takes place. A formula is suggested for calculation of the Reynolds number $R_{L k r}$, when the redistribution of the velocity profile takes place.

Substantiation of the suggested mechanism on momentum transfer in the boundary layer was made by putting together deduced calculation formulas and known in literature data, which gives fair accuracy.

The calculations show, that magnitudes of hydrodynamic characteristics are conditioned by variation behavior of the flow function.

It is also important to note, that within the large range $0<R e_{L}<10000$ of the Reynolds number variation the fluid motion is described by the same (1) and (2), without including the notion of turbulent viscosity.

\section{References}

[1] Schlichting, H., Gersten, K. (2017). Boundary-Layer Theory. Springer, 805. doi: https://doi.org/10.1007/978-3-662-52919-5

[2] Loitsyanskii, L. G. (1966). Mechanics of liquids and gases. Pergamon. doi: https://doi.org/10.1016/c2013-0-05328-5

[3] Anderson, D. A., Tannehill, J. C., Pletcher, R. H., Ramakanth, M., Shankar, V. (2020). Computational Fluid Mechanics and Heat Transfer. Boca Raton, 974. doi: https://doi.org/10.1201/9781351124027

[4] Repik, E. U., Sosedko, Yu. P. (2007). Turbulent boundary layer. Moscow: Fizmatgiz, 312. Available at: https://www.studmed.ru/ repik-eu-sosedko-yup-turbulentnyy-pogranichnyy-sloy-metodika-i-rezultaty-eksperimentalnyh-issledovaniy_de3b3744f70.html

[5] Sarukhanyan, A., Vartanyan, A., Vermishyan, G., Tokmajyan, V. (2020). The Study of Hydrodynamic Processes Occurring on Transition of Sudden Expanding of Hydraulic Section of Plane - Parallel Full Pipe Flow. TEM Journal, 9 (4), $1494-1501$. doi: https://doi.org/10.18421/tem94-23

[6] Sarukhanyan, A. A., Vartanyan, A. A., Vartanyan, G. G., Tokmajyan, H. V. (2021). Estimation of hydraulic structures safety by comparison of strength and stability theories. IOP Conference Series: Earth and Environmental Science, 677 (4), 042085. doi: https://doi.org/10.1088/1755-1315/677/4/042085

[7] Tokmajyan, H. V. (2016). On Movement of Suspended Particles in Turbulent Flow. Bulletin of High Technology, 1, 3-10. Available at: http://bulletin.am/wp-content/uploads/2016/10/Tokmajyan.pdf

[8] Lykov, A. V. (1978). Heat-and-mass transfer. Moscow: Energia, 480. Available at: https://www.studmed.ru/lykov-av-teplomassoobmen_44a57897b35.html

[9] Pavelyev, A. A., Reshmin, A. I., Teplovodskii, S. K., Fedoseev, S. G. (2003). On the lower critical Reynolds number for flow in a circular pipe. Fluid dynamics, 38 (4), 545-551. doi: https://doi.org/10.1023/a:1026369727130

[10] Kutateladze, S. S. (1982). Analysis of similarity in thermal physics. Novosibirsk: Science, 280. Available at: https://www. studmed.ru/kutateladze-s-s-analiz-podobiya-v-teplofizike_94ddb3da811.html

[11] Nadaoka, K., Hino, M., Koyano, Y. (1989). Structure of the turbulent flow field under breaking waves in the surf zone. Journal of Fluid Mechanics, 204, 359-387. doi: https://doi.org/10.1017/s0022112089001783 
[12] Corino, E. R., Brodkey, R. S. (1969). A visual investigation of the wall region in turbulent flow. Journal of Fluid Mechanics, 37 (1), 1-30. doi: https://doi.org/10.1017/s0022112069000395

[13] Belotserkovskii, O. M. (2009). Constructive modeling of structural turbulence and hydrodynamic instabilities. Singapore: World Scientific, 488. doi: https://doi.org/10.1142/6945

[14] Bykov, L. V., Molchanov, A. M., Yanyshev, D. S., Platonov, I. M. (2018). Current approaches to calculation of flow characteristics in laminar-turbulent transfer at boundary layer. Thermophysics of high temperatures, 56 (1), 104-120. doi: https://doi.org/10.7868/s0040364418010131

[15] Gorbushin, A. R., Zametev, V. B. (2017). Asymptotic analysis of viscous pulsation in turbulent boundary layer. Materials of the XXVIII Scientific and Technical Conference on Aerodynamics, 102. Available at: https://www.elibrary.ru/ item.asp?id=32626912

[16] Aktershev, S. P., Bartashevich, M. V., Chinnov, E. A. (2017). A semi analytic solution method for heat transfer in a fluid film in condition of constant heat stream on a wall. Thermophysics of high temperatures, 55 (1), 115-121. doi: https://doi.org/ $10.7868 / \mathrm{s} 004036441701001 \mathrm{x}$

[17] Vigdorovich, I. I. (2016). Reynolds analogy and a new formulation of temperature defect law for turbulent boundary on a plate. Reports of the USSR Academy of Sciences, 466 (4), 412-417. doi: https://doi.org/10.7868/s0869565215360128

[18] Laptinski, V. N. (2013). On an analytic method of a problem solution on dynamic laminar boundary layer in an auto model case. Scientific notes TsAGI, 44 (5), 72-93. Available at: https://cyberleninka.ru/article/n/ob-odnom-analiticheskom-metoderesheniya-zadachi-o-dinamicheskom-laminarnom-pogranichnom-sloe-v-avtomodelnom-sluchae/viewer

[19] Bird, R. B., Stewart, W. E., Lightfoot, E. N. (1974). Transport phenomena. Moscow: Himiya, 692. Available at: https://sng1lib. org/book/2397655/700109?id=2397655\&amp;secret=700109

[20] Nagasaki, T., Akiyama, H., Nakagawa, H. (2002). Numerical analysis of flow and mass transfer in a falling liquid film with interfacial waves. Thermal Science and Engineering, 10 (1), 17-23. Available at: https://ci.nii.ac.jp/naid/10010165308/

[21] Chinnov, E. A., Abdurakipov, S. S. (2013). Thermal entry length in falling liquid films at high Reynolds numbers. International Journal of Heat and Mass Transfer, 56 (1-2), 775-786. doi: https://oi.org/10.1016/j.ijheatmasstransfer.2012.08.058

[22] Sarkisyan, N. M. (2010). On mass transfer in fluid film, dripping over surface of vertically installed pipe. Chemical technology, 11 (3), 169-175. Available at: https://www.elibrary.ru/item.asp?id = 15277003

[23] Mellor, G. L. (1972). The large reynolds number, asymptotic theory of turbulent boundary layers. International Journal of Engineering Science, 10 (10), 851-873. doi: https://doi.org/10.1016/0020-7225(72)90055-9

[24] Demikhin, E. A., Kalaidin, E. N., Rastaturin, A. A. (2005). Influence of wave modes on mass transfer in flowing liquid films. Thermophysics and aeromechanics, 12 (2), 259-269. Available at: https://sibran.ru/upload/iblock/3f2/3f289e08e643edff72c8dd32af334463.pdf

How to cite: Sarukhanyan, A., Sarkisyan, N., Tokmajyan, V., Vartanyan, A. (2021). Study of fluid layer gravity motion over vertical surface. EUREKA: Physics and Engineering, 6, 28-38. doi: https://doi.org/10.21303/2461-4262.2021.002115 